\title{
Umbilical Cord Blood
}

National Cancer Institute

\section{Source}

National Cancer Institute. Umbilical Cord Blood. NCI Thesaurus. Code C13300.

Blood present in the umbilical vessels at the time of delivery. If cryopreserved at birth, cord blood can serve as a source of hematopoietic progenitor cells for transplantation to a patient later diagnosed and treated for a hematopoietic disorder. 